\title{
Upcoming support for triple stellar systems in PHOEBE
}

\author{
K.E. Conroy \\ Dept. of Astrophysics and Planetary Sciences, 800 E Lancaster Ave, Villanova \\ PA 19085, USA, (E-mail: kyle.conroy@villanova.edu)
}

Received: November 1, 2019; Accepted: November 24, 2019

\begin{abstract}
Eclipsing binary stars allow for the direct measurement of stellar parameters and distances and are therefore an important tool in the calibration of stellar relationships. In benchmark cases, we can achieve a precision of $2-3 \%$ in fundamental stellar parameters. Due to tighter constraints caused by mutual eclipse events, systems with additional companions allow achieving precision as low as $0.5 \%$. Triple systems have also been proposed as a mechanism for explaining an overabundance of short-period tight binaries. Despite all of this, we do not yet have a complete model for these multiple star systems that include tight binaries. In order to precisely and accurately model these complex systems, we must take into account several considerations, including: light time effects, perturbations to orbital elements, and the distortion of the stellar surfaces. Including all of these into a comprehensive treatment of triple and higher order systems within PHOEBE is currently under development and planned for an upcoming release.
\end{abstract}

\section{Introduction}

Detailed modeling of benchmark eclipsing binary systems can result in an accuracy of fundamental parameters (masses, radii, and temperatures) of $2-3 \%$ for $\sim 100$ main-sequence (Torres et al., 2010) and 15 pre-main-sequence stars (Stassun et al., 2014). These benchmark systems are used to callibrate a number of stellar models and relationships, and often our ability to discriminate between models is being limited by this precision. For example, Torres et al. (2010) shows a scatter in the luminosity-mass relationship that is larger than the uncertainties on the individual systems. This variation is likely due to varying evolutionary stages and metallicities among the population, but in order to better understand these trends we need to model more eclipsing binary systems and continue to push the limits of precision.

We can take several steps towards improving the precision and accuracy in modeled parameters of eclipsing binary systems. In recent years, photometric precision has seen significant improvement from Kepler and other space-based missions. In order to handle this increase in observational precision, we have actively been working to improve our models to account for higher-order effects, 
remove assumptions, and minimize computational noise. These improvements in both observation and model precision now allow us to approach the $1 \%$ level in fundamental parameters (Prša et al., 2016; Horvat et al., 2018). But ultimately, we are still limited by the inherent degeneracies - caused by the highly non-linear and correlated parameter space - of these systems which provide a "floor" on the achievable precision. For example, the width of an eclipse can be increased by increasing the radius of either star or slightly increasing the orbital inclination, resulting in a degeneracy in the model which limits our ability to achieve better precision in these fundamental parameters.

In certain cases, adding a third star into the system allows for breaking some of these inherent degeneracies and therefore allows for directly measuring these fundamental parameters to a precision an order of magnitude better than for eclipsing binary systems without companions.

\section{Benefits to modeling triple systems}

Triple (and higher-order multiple systems) allow for much more stringent constraints on these fitted fundamental parameters - by as much as an order of magnitude improvement over binary systems, finally breaching into sub-percent uncertainties (cf. Carter et al., 2011; Doyle et al., 2011; Welsh et al., 2012). Imagine a third star crossing in front of the inner-binary with the degeneracy between radius and inclination - the exact timing and shape of the resulting eclipse places stringent geometric constraints that break the previous degeneracies, resulting in a unique solution with unprecedented uncertainties (see KOI-126 in Carter et al. 2011 for an example).

In addition to this increased precision, the presence of additional bodies enables us to study and test the effects of their mutual gravitational influence. For example, eclipsing binaries in large photometric surveys exhibit a peak in the orbital period distribution on the order of 1 day (Devor, 2005; Paczyński et al., 2006; Derekas et al., 2007). Binary formation theory, however, struggles to explain the creation of these close binaries in situ, and capture seems unlikely to produce the large number of observed close binaries (Bonnell, 2001). KozaiLidov Cycles and Tidal Friction (KCTF) have been proposed as a formation mechanism for these short-period binaries through the interaction with a third star on an eccentric and inclined orbit with respect to the inner-binary (Kozai, 1962; Lidov, 1962; Fabrycky \& Tremaine, 2007; Naoz et al., 2013). Fabrycky \& Tremaine (2007) used simulations of systems undergoing KCTF to provide testable distributions in the final mutual inclination between the resulting close inner-binaries and their companions. Other methods including disk migration have also been proposed to accomplish the same result of tight inner-binaries, resulting in a testable difference in the expected distributions of orbital parameters. In order to test these theories, we need to accurately and precisely model a large number of observed triple systems, specifically those containing tight 
inner-binaries, so that we can compare these theoretical distributions against observations.

Tokovinin et al. (2006) found that $60 \%$ of binaries with orbital periods less than 3 days have a tertiary component, compared to only $34 \%$ of binaries with periods greater than 12 days. Many of these short-period tight binaries could have tertiary components on wide or inclined orbits, making the triple nature difficult to detect in the light curves, radial velocity curves, or eclipse timing variations. Searches of eclipse timing variations among Kepler eclipsing binary systems (Prša et al., 2011; Slawson et al., 2011; Kirk et al., 2016), show that about $15-20 \%$ of short-period binaries show evidence of tertiary components within the $\sim 4$ year time-baseline of Kepler (Gies et al., 2012; Rappaport et al., 2013; Conroy et al., 2014). This still leaves a large number of tight binaries in which the expected tertiary component cannot easily be detected in the light curve or eclipse timing variations. The dynamics of these distorted eclipsing binaries may still be subject to the effects of a tertiary companion, even if that companion is not detected directly.

For all of these reasons, it is necessary to develop a code that can robustly handle triple and higher-order systems, including all the advanced high-order effects required to adequately model Kepler-like photometry, as well as both dynamical and surface distortion effects.

\section{Future implementation in PHOEBE}

To address the need to model these systems, we plan to include support for generic hierarchies with any number of components into the PHOEBE ${ }^{1}$ eclipsing binary software package (Prša \& Zwitter, 2005; Prša et al., 2016; Horvat et al., 2018).

We plan to allow for flexible generic hierarchies and parameterization, such that any system can be defined through any number of nested Keplerian orbits. Although the systems would be defined in this strictly hierarchical manner, the dynamics would then be handled through an n-body integrator initialized by the Keplerian orbits at some time, $t_{0}$. As is currently the case for binaries with Keplerian orbits, the position of each component would be offset in its orbit such that its light reaches the barycenter of the system at any requested observation time - effectively handling any light travel time effects.

In order to handle the tight inner-binaries in higher-order systems, it is important to have the capability to handle surface distortion, rather than falling back on the spherical assumption for all the components in the system. To do this, we plan to use the n-body dynamics to determine instantaneous Keplerian representations of the current position of each individual component at any given time. From the Keplerian elements (period, semi-major axis, eccentricity), we can then use the Roche framework to determine the distortion of each star

${ }^{1}$ http://phoebe-project.org 
caused by its "sibling" in the hierarchy using the same assumption of instantaneously adapting surfaces to conserve volume currently used in the two-body scenario. This "hybrid" approach allows for a model that accounts for both the dynamical interaction between multi-bodies, as well as the surface distortion caused by tight pairs of stars and the resulting effects on the observables.

By implementing support for any higher order system into PHOEBE, we will have a code capable of robustly modeling these systems taking advantage of all the higher-order effects incorporated into the code for binary systems. However, this all comes at computational cost. In many cases (particularly for stable systems without dynamical effects or those where surface distortion can be ignored), existing codes are fully capable and may be significantly cheaper. We hope to provide wrapper convenience functions for these other codes - both to make it easier to compare the outputs of the codes but also to use the backend that is most efficient given the restrictions and valid assumptions for any particular system.

Acknowledgements. PHOEBE is made possible through NSF 1517474 and NASA 17-ADAP17-68, which we gratefully acknowledge.

\section{References}

Bonnell, I. A., The Formation of Close Binary Stars. 2001, in IAU Symposium, Vol. 200, The Formation of Binary Stars, ed. H. Zinnecker \& R. Mathieu, 23

Carter, J. A., Fabrycky, D. C., Ragozzine, D., et al., KOI-126: A Triply Eclipsing Hierarchical Triple with Two Low-Mass Stars. 2011, Science, 331, 562, DOI: 10.1126/science. 1201274

Conroy, K. E., Prša, A., Stassun, K. G., et al., Kepler Eclipsing Binary Stars. IV. Precise Eclipse Times for Close Binaries and Identification of Candidate Threebody Systems. 2014, Astron. J., 147, 45, DOI: 10.1088/0004-6256/147/2/45

Derekas, A., Kiss, L. L., \& Bedding, T. R., Eclipsing Binaries in the MACHO Database: New Periods and Classifications for 3031 Systems in the Large Magellanic Cloud. 2007, Astrophys. J., 663, 249, DOI: 10.1086/517994

Devor, J., Solutions for 10,000 Eclipsing Binaries in the Bulge Fields of OGLE II Using DEBiL. 2005, Astrophys. J., 628, 411, DOI: 10.1086/431170

Doyle, L. R., Carter, J. A., Fabrycky, D. C., et al., Kepler-16: A Transiting Circumbinary Planet. 2011, Science, 333, 1602, DOI: 10.1126/science.1210923

Fabrycky, D. \& Tremaine, S., Shrinking Binary and Planetary Orbits by Kozai Cycles with Tidal Friction. 2007, Astrophys. J., 669, 1298, DOI: 10.1086/521702

Gies, D. R., Williams, S. J., Matson, R. A., et al., A Search for Hierarchical Triples using Kepler Eclipse Timing. 2012, Astron. J., 143, 137, DOI: 10.1088/0004$6256 / 143 / 6 / 137$ 
Horvat, M., Conroy, K. E., Pablo, H., et al., Physics of Eclipsing Binaries. III. SpinOrbit Misalignment. 2018, Astrophys. J., Suppl., 237, 26, DOI: 10.3847/1538$4365 /$ aacd0f

Kirk, B., Conroy, K., Prša, A., et al., Kepler Eclipsing Binary Stars. VII. The Catalog of Eclipsing Binaries Found in the Entire Kepler Data Set. 2016, Astron. J., 151, 68, DOI: 10.3847/0004-6256/151/3/68

Kozai, Y., Secular perturbations of asteroids with high inclination and eccentricity. 1962, Astron. J., 67, 591, DOI: 10.1086/108790

Lidov, M. L., The evolution of orbits of artificial satellites of planets under the action of gravitational perturbations of external bodies. 1962, Planet. Space Sci., 9, 719, DOI: 10.1016/0032-0633(62)90129-0

Naoz, S., Farr, W. M., Lithwick, Y., Rasio, F. A., \& Teyssandier, J., Secular dynamics in hierarchical three-body systems. 2013, Mon. Not. R. Astron. Soc., 431, 2155, DOI: $10.1093 / \mathrm{mnras} / \mathrm{stt} 302$

Paczyński, B., Szczygieł, D. M., Pilecki, B., \& Pojmański, G., Eclipsing binaries in the All Sky Automated Survey catalogue. 2006, Mon. Not. R. Astron. Soc., 368, 1311, DOI: $10.1111 / \mathrm{j} .1365-2966.2006 .10223 . \mathrm{x}$

Prša, A., Batalha, N., Slawson, R. W., et al., Kepler Eclipsing Binary Stars. I. Catalog and Principal Characterization of 1879 Eclipsing Binaries in the First Data Release. 2011, Astron. J., 141, 83, DOI: 10.1088/0004-6256/141/3/83

Prša, A., Conroy, K. E., Horvat, M., et al., Physics Of Eclipsing Binaries. II. Toward the Increased Model Fidelity. 2016, Astrophys. J., Suppl., 227, 29, DOI: $10.3847 / 1538-4365 / 227 / 2 / 29$

Prša, A. \& Zwitter, T., A Computational Guide to Physics of Eclipsing Binaries. I. Demonstrations and Perspectives. 2005, Astrophys. J., 628, 426, DOI: $10.1086 / 430591$

Rappaport, S., Deck, K., Levine, A., et al., Triple-star Candidates among the Kepler Binaries. 2013, Astrophys. J., 768, 33, DOI: 10.1088/0004-637X/768/1/33

Slawson, R. W., Prša, A., Welsh, W. F., et al., Kepler Eclipsing Binary Stars. II. 2165 Eclipsing Binaries in the Second Data Release. 2011, Astron. J., 142, 160, DOI: 10.1088/0004-6256/142/5/160

Stassun, K. G., Feiden, G. A., \& Torres, G., Empirical tests of pre-main-sequence stellar evolution models with eclipsing binaries. 2014, NewAR, 60, 1, DOI: 10.1016/j.newar.2014.06.001

Tokovinin, A., Thomas, S., Sterzik, M., \& Udry, S., Tertiary companions to close spectroscopic binaries. 2006, Astron. Astrophys., 450, 681, DOI: 10.1051/00046361:20054427

Torres, G., Andersen, J., \& Giménez, A., Accurate masses and radii of normal stars: modern results and applications. 2010, Astron. Astrophys. Rev., 18, 67, DOI: $10.1007 / \mathrm{s} 00159-009-0025-1$

Welsh, W. F., Orosz, J. A., Carter, J. A., et al., Transiting circumbinary planets Kepler-34 b and Kepler-35 b. 2012, Nature, 481, 475, DOI: 10.1038/nature10768 